\title{
RESPOSTA DE PACIENTES HIPERTENSOS SOB TRATAMENTO MEDICAMENTOSO DE ACORDO COM OS NÍVEIS PRESSÓRICOS
}

\author{
Ludmilla Maria de Oliveira e OLIVEIRA, Guilherme Barbosa de Souza ARAÚJO, Jorge Otávio Gonçalves \\ FERREIRA, Vinícius Gonçalves FERRAS, Carlos Rubens Ponchini CARVALHO \& Denise Aparecida da \\ SILVA*
}

1 Universidade Iguaçu - Campus V. Itaperuna, Rio de Janeiro, Brasil.

*Autor para correspondência: dearasp@yahoo.com.br

DOI: http://dx.doi.org/10.18571/acbm.186

\section{RESUMO}

Introdução: Análises pregressas evidenciam o baixo percentual de controle da pressão arterial (PA) em indivíduos hipertensos sob tratamento medicamentoso. O objetivo desta pesquisa foi avaliar a PA de pacientes sob tratamento farmacológico. Metodologia: A pesquisa foi realizada nas Unidades de Saúde da Família do município de Itaperuna - RJ., durante os meses de fevereiro a julho de 2017. Resultados: Foram avaliados 215 pacientes (60\% do sexo feminino e $40 \%$ do sexo masculino) com idade média de 59,79 $\pm 12,64$ anos sendo $61,86 \%$ brancos, $24,19 \%$ negros e $13,95 \%$ pardos. A maioria possuía ensino fundamental incompleto $(39,07 \%)$ e se encontrava na classe D $(37,21 \%)$ conforme a classificação socioeconômica. A maioria dos pacientes $(62,33 \%)$ encontrava-se sob tratamento com mais de um medicamento anti-hipertensivo. As principais classes de medicamentos anti-hipertensivos verificadas no tratamento dos pacientes incluíram os betabloqueadores $(46,51 \%)$ seguidos dos BRA (40,93\%) e diuréticos (39,53\%). Os níveis pressóricos dos pacientes variaram de 110 a $220 \mathrm{mmHg}$ para a PAS e de 60 a $140 \mathrm{mmHg}$ para a PAD, sendo que $33,02 \%$ dos pacientes apresentaram níveis pressóricos superiores ao limite de normalidade. Conclusões: Os medicamentos mais usados no tratamento e controle da HAS não são os mais eficazes atualmente e um número expressivo de pacientes sob tratamento não apresenta níveis pressóricos normalizados, em especial aqueles sob tratamento com dois medicamentos antihipertensivos. A pesquisa deixa clara a necessidade de melhor acompanhamento dos pacientes pelos profissionais de saúde quanto à resposta à terapia medicamentosa a fim de que se possa realmente prevenir danos maiores em órgãos-alvo.

Palavras-chave: Hipertensão arterial; Anti-hipertensivos; Monoterapia, Politerapia.

\begin{abstract}
Introduction: Preliminary analyzes show the low percentage of blood pressure control (BP) in hypertensive individuals under drug treatment. The objective of this research was to evaluate the BP of patients under pharmacological treatment. Methodology: The research was carried out in the Family Health Units of the city of Itaperuna - RJ, during the months of February to July of 2017. Results: It was analyzed a total of 215 patients (60\% female and $40 \%$ male) with a mean age of $59.79 \pm 12.64$ years, $61.86 \%$ white, $24.19 \%$ black and $13.95 \%$ brown. The majority had incomplete primary education $(39.07 \%)$ and was included in class D (37.21\%) according to socioeconomic classification. The majority of patients $(62.33 \%)$ were being treated with more than one antihypertensive drug. The main classes of antihypertensive drugs observed in the treatment of patients included beta-blockers $(46.51 \%)$ followed by angiotensin II receptor blockers $(40.93 \%)$ and diuretics (39.53\%). Patients pressure levels ranged from 110 to $220 \mathrm{mmHg}$ for systolic blood pressure and from 60 to $140 \mathrm{mmHg}$ for diastolic blood pressure, with $33.02 \%$ of patients presenting blood pressure levels above the normal range. Conclusions: The most used drugs in the treatment and control of hypertension are not the most effective currently and an expressive number of the
\end{abstract}


patients undergoing treatment do not present normalized blood pressure levels, especially those under treatment with two antihypertensive drugs. The research makes clear the need for better follow-up of patients by health professionals regarding the response to drug therapy in order to actually prevent further damage to target organs.

Keywords: Hypertension; Antihypertensives; Monotherapy, Polytherapy.

\section{Introdução}

A Hipertensão arterial (HA) é uma condição clínica multifatorial caracterizada pela elevação sustentada dos níveis pressóricos $\geq 140$ e/ou $90 \mathrm{mmHg}$ para as pressões sistólica e diastólica, respectivamente. No Brasil, a HA atinge 32,5\% (36 milhões) de indivíduos adultos, mais de $60 \%$ dos idosos e contribui direta ou indiretamente para $50 \%$ das mortes por doença cardiovascular (DCV). Os estudos referentes à prevalência divergem de acordo com a população estudada e o método de avaliação, apresentando-se em torno de $27 \%$ em homens e mulheres, com poucas diferenças, havendo uma associação direta e linear com o envelhecimento (MALACHIAS et al., 2016). A HA está diretamente relacionada a fatores de risco modificáveis e não modificáveis, tais como: idade, gênero, etnia, hábitos de vida, tabagismo, ingestão de sal, ingestão de álcool, sedentarismo e excesso de peso. Estudos indicam que os fatores de risco mais prevalentes são sedentarismo (39,31\%), sobrepeso $(32,09 \%)$ e tabagismo $(22,84 \%)$. A manutenção dos altos níveis pressóricos apresenta como consequências a diminuição da expectativa e qualidade de vida, uma vez que é considerado fator predisponente de arteriosclerose e trombose que, por sua vez, estão relacionados a quadros de isquemia cardíaca, cerebral e vascular (CARVALHO et al., 2013; MENEZES et al., 2017).

O tratamento é baseado na associação de medicamentos anti-hipertensivos com medidas não farmacológicas, sendo ambos fundamentais para o controle da pressão arterial (PA) e dos riscos associados. O tratamento terapêutico medicamentoso destina-se não apenas à redução dos níveis pressóricos, mas fundamentalmente na prevenção de eventos cardiovasculares fatais e não fatais, bem como na redução da taxa de mortalidade. Estimativas mostram que dois terços dos acidentes vasculares encefálicos e $50 \%$ das doenças coronarianas possam ser atribuídos a níveis pressóricos elevados (IBRAHIM; DAMASCENO; 2012; MALACHIAS et al., 2016).

As principais classes de anti-hipertensivos disponíveis para uso clínico são os diuréticos (tiazídicos, de alça e poupadores de potássio), inibidores adrenérgicos (de ação central, betabloqueadores e alfa bloqueadores), vasodilatadores diretos, bloqueadores dos canais de cálcio (BCC), inibidores da enzima conversora de angiotensina (IECA), bloqueadores do receptor AT1 da angiotensina II (BRA) e inibidor direto da renina. Além dos benefícios clínicos, outros fatores essenciais para o sucesso do tratamento devem ser considerados, tais como morbidades associadas à HAS, efeitos adversos, potenciais interações medicamentosas, custo e acessibilidade (DUARTE et al., 2014). O tratamento não medicamentoso consiste em estratégias que visam mudar o estilo de vida e que possam levar à diminuição da dosagem dos medicamentos ou até mesmo à sua retirada do tratamento. Apesar da existência dos diversos tratamentos previamente citados, apenas $1 / 3$ dos pacientes adultos conseguem adequado controle pressórico. Tal fato está associado em especial à baixa adesão ao tratamento medicamentoso que pode ocorrer por diversos motivos, tais como efeitos colaterais das medicações, esquecimento do uso, custo da medicação, receio de misturar com álcool, desconhecimento da necessidade de continuidade do tratamento, uso de tratamento alternativo, medo de intoxicação e hipotensão e medo de misturar com outras drogas. Dados americanos epidemiológicos demonstram que dentre $54 \%$ das pessoas que sabem ser hipertensas e recebem tratamento para esta condição, apenas $27 \%$ têm a sua PA controlada dentro dos níveis recomendados (ANDRADE et al., 2002). 
A adesão ao tratamento da HAS é de fundamental importância para o controle da doença, em contrapartida, é um dos maiores desafios no seu combate. A adesão ao tratamento corresponde à concordância entre a prescrição médica e a conduta entendida pelo próprio paciente. Porém, são muitos os fatores que contribuem para a não adesão ao tratamento, que vão além do simples seguimento da prescrição de medicamentos e envolvem aspectos referentes ao sistema de saúde, fatores socioeconômicos, além de aspectos relacionados ao tratamento, paciente e à própria doença (GUSMÃO; MION JR, 2006; GIROTTO et al., 2013).

Considerando-se que análises pregressas evidenciam o baixo percentual de controle da PA para indivíduos diagnosticados como hipertensos, esta pesquisa apresentou como objetivo avaliar os níveis pressóricos de pacientes sob tratamento farmacológico, a fim de avaliar a resposta ao tratamento medicamentoso conforme os níveis pressóricos. O controle adequado dos níveis pressóricos reduz a progressão da doença e, assim, aumenta a sobrevida dos pacientes bem como reduz os gastos pessoais e dos sistemas públicos de saúde.

\section{Metodologia}

Uma pesquisa de origem casuística, qualitativa e quantitativa foi realizada nas Unidades de Saúde da Família do município de Itaperuna - RJ, durante os meses de fevereiro a julho de 2017. Atualmente, o município conta com um total de 21 unidades ou Postos de saúde da Família distribuídos pelos diversos bairros. Como critério de inclusão, participaram da pesquisa os pacientes atendidos com idade igual ou superior a 18 anos, com quadro de HA diagnosticado e sob tratamento medicamentoso e que concordaram e assinaram um Termo de Consentimento Livre e Esclarecido, conforme Resolução nº 466 de 12 de dezembro de 2012 (BRASIL, 2013). Os pacientes foram submetidos a exame para aferição da PA pelo método indireto, com técnica auscultatória e uso do esfigmomanômetro do tipo aneroide com graduação até $300 \mathrm{mmHg}$, sendo a aferição realizada por três vezes contando-se com período inicial de repouso de cinco minutos, de acordo com recomendações das Diretrizes Brasileiras de Hipertensão Arterial (Sociedade Brasileira de Cardiologia; Sociedade Brasileira de Hipertensão; Sociedade Brasileira de Nefrologia, 2010). Aos pacientes foi também aplicado um questionário referente aos aspectos pessoais, visando ao melhor conhecimento do seu perfil, bem como ao tratamento medicamentoso a eles prescrito. Previamente à pesquisa foi disponibilizado um termo de autorização aos responsáveis para a realização da pesquisa nas unidades de saúde. Os resultados foram avaliados através da aplicação de estatística descritiva.

\section{Resultados}

No total foram avaliados 215 pacientes, sendo 129 do sexo feminino (60\%) e 86 do sexo masculino (40\%), conforme figura 1. A idade dos pacientes variou de 28 a 89 anos com média de $59,79 \pm 12,64$ anos, considerando-se ambos os sexos e, quanto à etnia, 133 eram brancos, 52 negros e 30 pardos, perfazendo $61,86 \%, 24,19 \%$ e $13,95 \%$, respectivamente. 


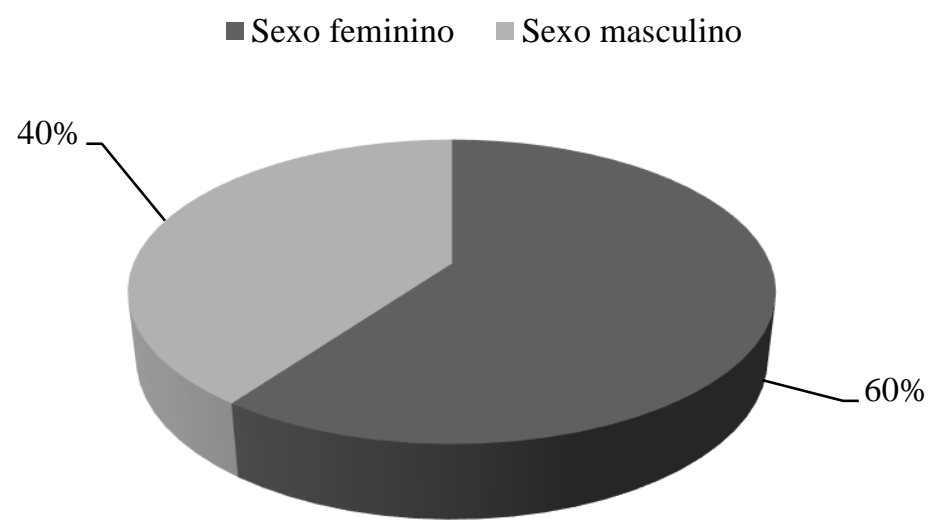

Figura 1. Percentual de pacientes hipertensos de acordo com o sexo, conforme pesquisa realizada em Unidades Básicas de Saúde do município de Itaperuna, RJ.

Considerando-se a classificação dos pacientes quanto ao grau de escolaridade, a maioria possuía ensino fundamental incompleto $(39,07 \%)$ sendo $6,51 \%$ analfabetos. De acordo com a classificação econômica, a maioria $(37,21 \%)$ encontrava-se na classe D e nenhum nas classes A e B. Os dados referentes ao grau de escolaridade e classificação econômica encontram-se na tabela 1 .

Tabela 1: Grau de escolaridade, etnia e classificação econômica de pacientes hipertensos, conforme pesquisa realizada em Unidades Básicas de Saúde do município de Itaperuna, RJ.

\begin{tabular}{lc}
\hline Informações pessoais & Número de pacientes \\
\hline - Etnia & $133(61,86 \%)$ \\
. Branco & $52(24,19 \%)$ \\
. Negro & $30(13,95 \%)$ \\
. Pardo & \\
- Grau de escolaridade & $14(6,51 \%)$ \\
. Analfabetos & $84(39,07 \%)$ \\
. Ensino fundamental incompleto & $23(10,70 \%)$ \\
. Ensino fundamental completo & $34(15,81 \%)$ \\
. Ensino médio incompleto & $46(21,40 \%)$ \\
. Ensino médio completo & $02(0,93 \%)$ \\
. Ensino superior incompleto & $12(5,58 \%)$ \\
. Ensino superior completo & \\
- Classificação econômica & - \\
. Classe A & - \\
. Classe B & $58(26,98 \%)$ \\
. Classe C & $80(37,21 \%)$ \\
. Classe D & $77(35,81 \%)$ \\
. Classe E &
\end{tabular}

Ao se avaliar os medicamentos em uso, a maioria $(62,33 \%)$ dos pacientes encontrava-se sob tratamento com mais de um medicamento anti-hipertensivo, sendo a monoterapia observada em $37,67 \%$ (Figura 2). 


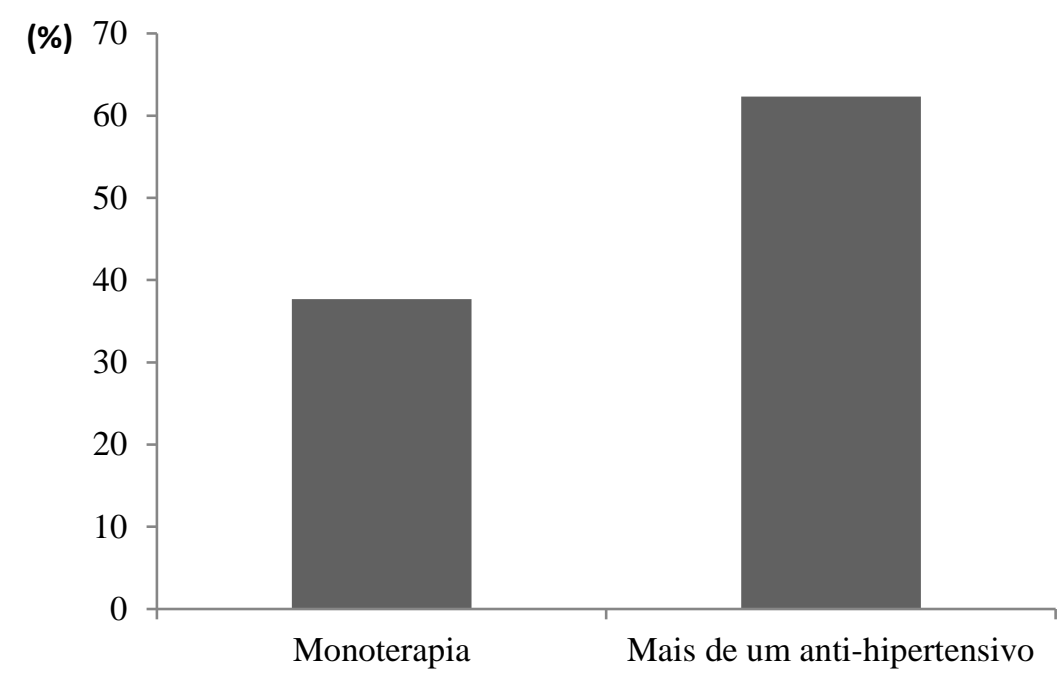

Figura 2: Número de pacientes hipertensos sob tratamento com medicamentos anti-hipertensivos em monoterapia ou não, conforme pesquisa realizada em Unidades Básicas de Saúde do município de Itaperuna, RJ.

As classes de medicamentos anti-hipertensivos verificadas no tratamento dos pacientes (monoterapia ou associados) incluíram os betabloqueadores com o maior número de pacientes $(46,51 \%)$ seguidos dos BRA (40,93\%) e dos diuréticos $(39,53 \%)$. Na figura 3 podem ser observadas as outras classes de medicamentos em uso.

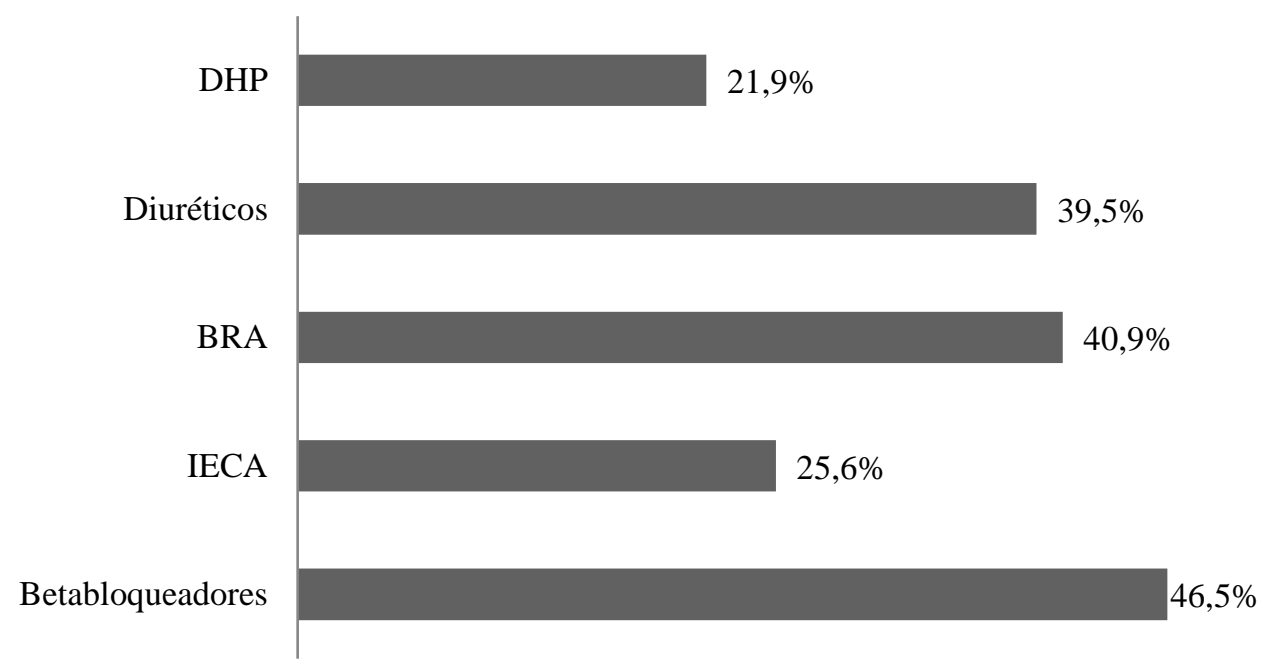

Figura 3: Percentual de pacientes sob tratamento de acordo com a classe de medicamentos anti-hipertensivos, conforme pesquisa realizada em Unidades Básicas de

Saúde do município de Itaperuna, RJ. Onde: BRA (bloqueadores dos receptores da angiotensina II), IECA (Inibidores da enzima conversora de angiotensina), DHP (bloqueadores de canais de cálcio dihidroiridínicos).

As classes de medicamentos anti-hipertensivos presentes no tratamento com monoterapia incluíram os IECA (presente no tratamento de $28,40 \%$ do total de pacientes sob monoterapia), seguidos dos betabloqueadores (25,92\%), BRA (24,69\%), diuréticos $(12,35 \%)$ e BCC $(8,64 \%)$, conforme figura 4 . Os medicamentos mais prescritos em cada classe foram o captopril, atenolol, losartana, hidroclorotiazida e anlodipino. 


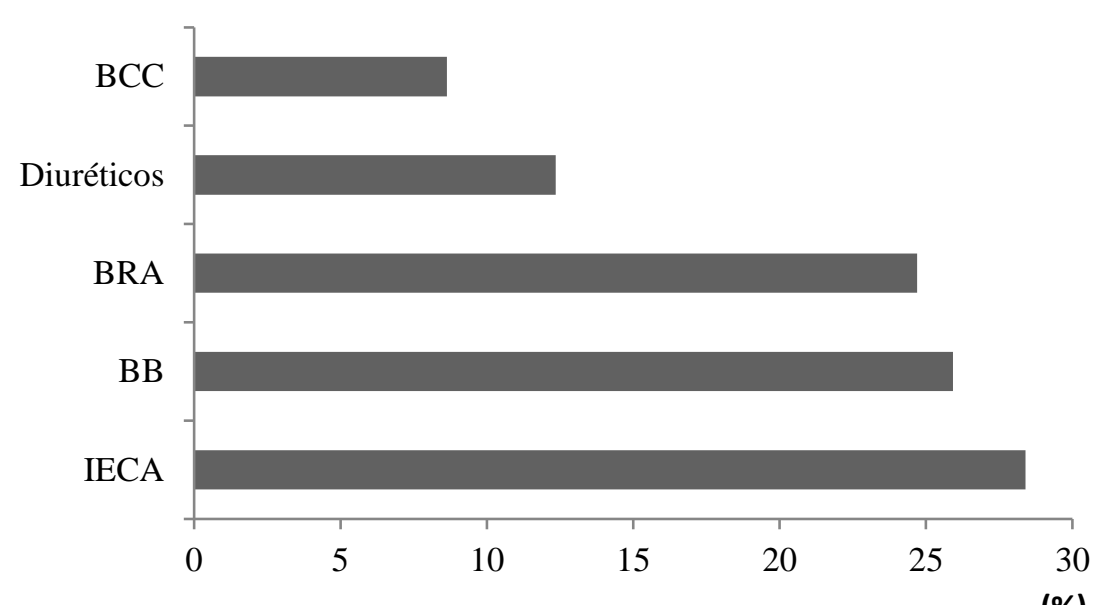

(\%)

Figura 4: Percentual de pacientes sob tratamento com monoterapia de acordo com a classe de medicamentos anti-hipertensivos, conforme pesquisa realizada em Unidades Básicas de Saúde do município de Itaperuna, RJ. Onde: IECA (Inibidores da enzima conversora de angiotensina); BB (Betabloqueadores); BRA (Bloqueadores dos receptores da angiotensina II), BCC (Bloqueadores de canais de cálcio).

Em se tratando das associações de anti-hipertensivos, foram observadas 105 contendo dois medicamentos, sendo as mais frequentes: diurético com betabloqueador $(23,8 \%)$, diurético com BRA $(18,1 \%)$ e betabloqueador com BRA $(16,2 \%)$, de acordo com figura 5.

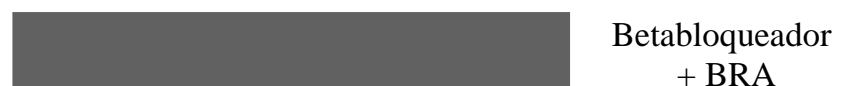

+ BRA
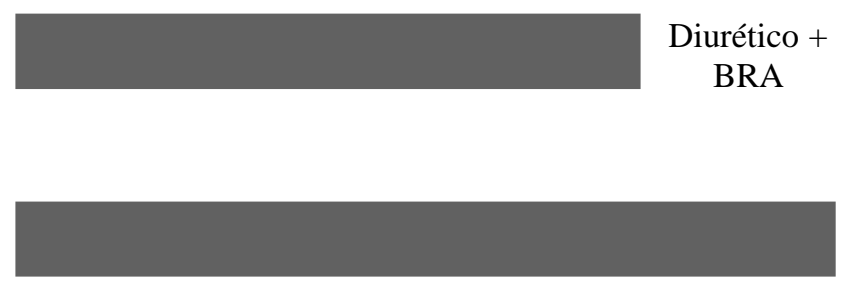

Diurético + betabloqueador

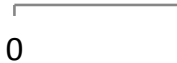

5

10

15

20

(\%)

Figura 5: Principais associações de anti-hipertensivos de pacientes sob tratamento com dois medicamentos, conforme pesquisa realizada em Unidades Básicas de Saúde do município de Itaperuna, RJ. Onde: BRA (bloqueadores dos receptores da angiotensina II).

As associações com três medicamentos faziam parte do protocolo de tratamento de 24 pacientes, sendo que em 70,8\% estava incluído um diurético. À avaliação geral dos medicamentos anti-hipertensivos, pôde-se verificar que os betabloqueadores ainda são os mais presentes nos protocolos de tratamento (46\%), seguidos dos BRA (40,9\%), diuréticos $(40,5 \%)$, IECA $(25,6 \%)$ e BCC $(20,9 \%)$, conforme figura 6. 


\section{Biomedica Brasiliensia}

ISSN: 2236-0867

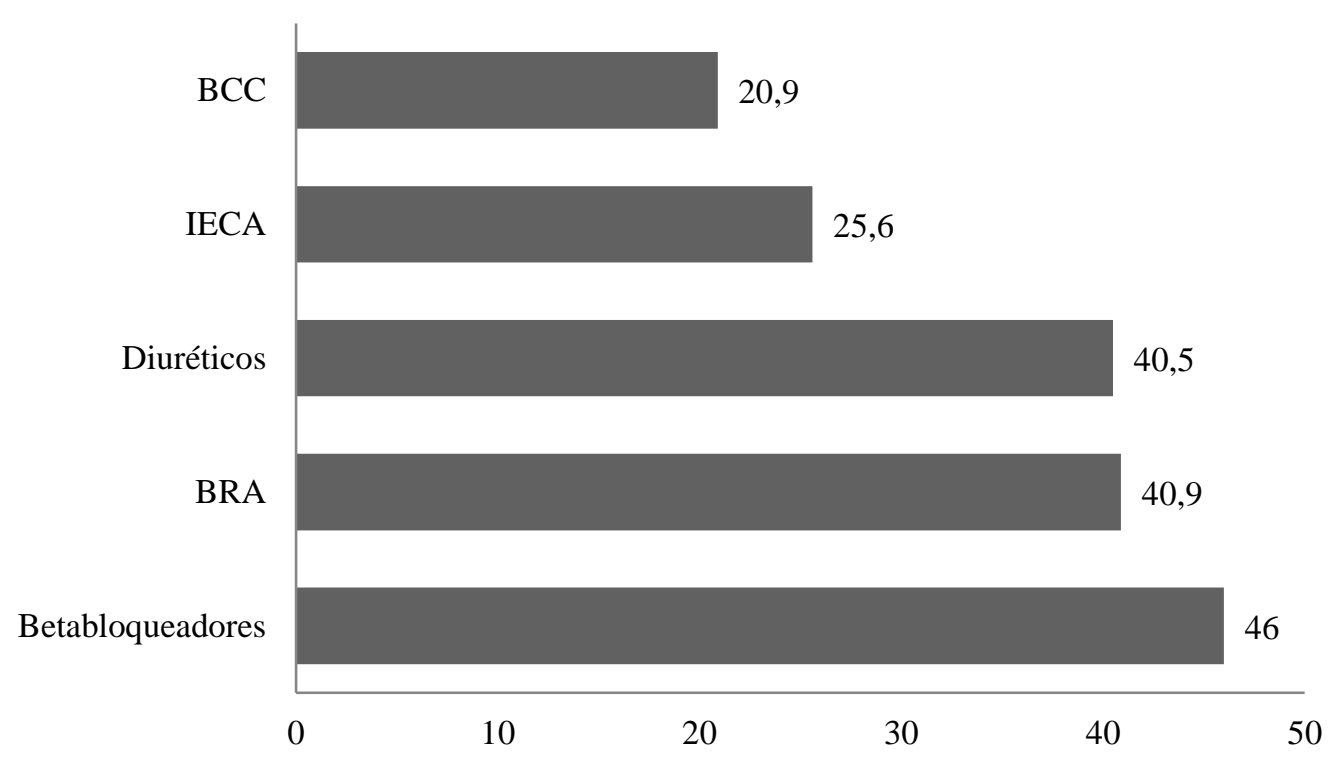

Figura 6: Principais anti-hipertensivos presentes nos protocolos de tratamento, conforme pesquisa realizada em Unidades Básicas de Saúde do município de Itaperuna, RJ. Onde:

BRA (bloqueadores dos receptores da angiotensina II), IECA (Inibidores da enzima conversora de angiotensina), BCC (bloqueadores de canais de cálcio).

A maioria dos pacientes encontrava-se sob tratamento há mais de 10 anos e os efeitos colaterais foram citados por $12,09 \%$ dos pacientes, incluindo tosse seca (sete pacientes), cansaço (seis pacientes), câimbras (três pacientes), edema (dois pacientes), cefaleia (dois pacientes), boca seca (dois pacientes), diarreia (dois pacientes), dor epigástrica (um paciente), náuseas (um paciente) e sonolência (um paciente). Vale ressaltar que vários pacientes se encontravam sob tratamento simultaneamente com outros medicamentos não anti-hipertensivos, o que justifica alguns dos efeitos colaterais verificados.

Sobre a associação também do tratamento não medicamentoso, 50,70\% dos pacientes afirmaram a não adesão, enquanto que $30,23 \%$ citaram que realizam o tratamento não medicamentoso associado e 19,07\% afirmaram que mais ou menos.

Os níveis pressóricos dos pacientes variaram de 110 a $220 \mathrm{mmHg}$ para a PAS e de 60 a $140 \mathrm{mmHg}$ para a PAD, sendo que 33,02\% dos pacientes apresentaram níveis pressóricos superiores ao limite de normalidade e $66,98 \%$ níveis pressóricos inferiores a $140 \mathrm{mmHg}$ para a PAS e $90 \mathrm{mmHg}$ para a PAD (Figura 7).

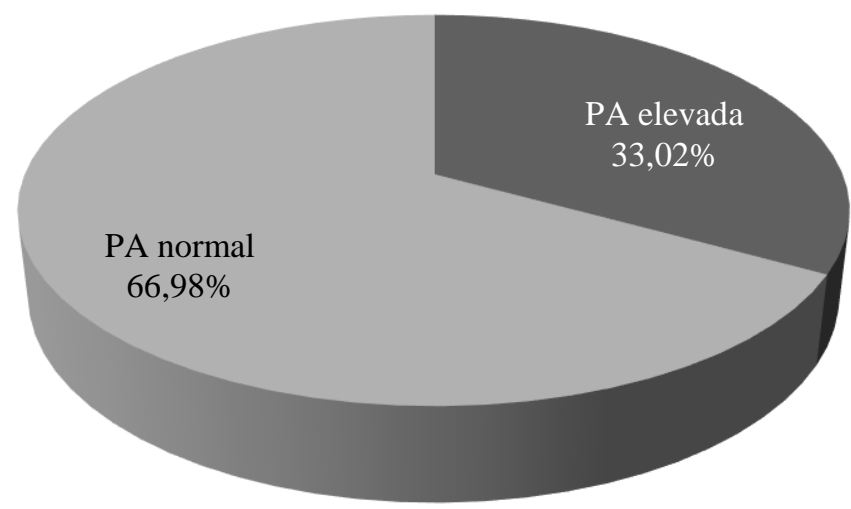

Figura 7: Níveis pressóricos de pacientes sob tratamento com anti-hipertensivos, conforme pesquisa realizada em Unidades Básicas de Saúde do município de Itaperuna, RJ. 
Considerando-se o número de medicamentos anti-hipertensivos utilizados no tratamento dos pacientes cuja PA estava elevada, 24,28\% estavam sob tratamento com monoterapia, 48,57\% com dois medicamentos anti-hipertensivos, $24,28 \%$ com três medicamentos e 2,86\% com mais de três medicamentos (Figura 8).
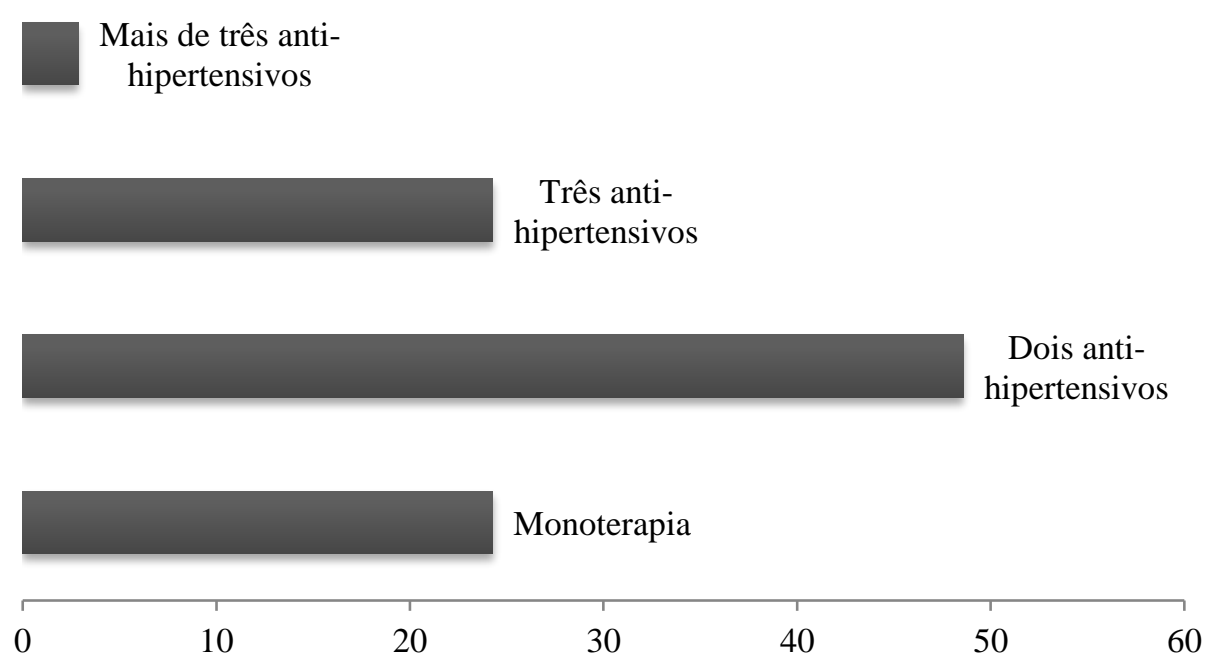

Figura 8: Percentual de pacientes com níveis pressóricos não controlados conforme o número de medicamentos anti-hipertensivos em uso, de acordo com pesquisa realizada em Unidades Básicas de Saúde do município de Itaperuna, RJ.

\section{Discussão}

A maioria dos pacientes que participou da pesquisa pertencia ao sexo feminino, resultado este já esperado e em concordância com outras pesquisas realizadas (BRITO VIEIRA et al., 2016) uma vez que as mulheres, geralmente, procuram mais pelos serviços de saúde e apresentam maior preocupação em se tratando de doenças o que, segundo Pimenta et al. (2015), também contribui para o diagnóstico mais precoce das doenças e seu tratamento. A idade média dos pacientes encontra-se de acordo com várias pesquisas tais como de Sudré et al. (2014), realizada no Brasil, que revelou a predominância de idosos hipertensos na faixa etária de 60 a 69 anos. Os baixos níveis de escolaridade e socioeconômicos verificados na pesquisa para a maioria dos pacientes hipertensos concordam com Barros et al. (2011) assim como com Reis e Cesarino (2014), respectivamente. A baixa escolaridade é associada, segundo os autores citados, ao fato de que a maioria dos idosos na atualidade não apresentou oportunidades de estudo quando mais jovens.

Os resultados referentes ao número de anti-hipertensivos em uso estão de acordo com diversos autores que citam a politerapia como frequente nos pacientes com HA. No entanto, foi possível observar que mais de $50 \%$ dos pacientes não apresenta adesão ao tratamento não medicamentoso, o que pode estar relacionado à menor resposta pressórica com o uso de medicamentos, levando à necessidade de associação. Somente a redução do peso corporal pode promover a redução de até $10 \mathrm{mmHg}$ nos níveis pressóricos, conforme o peso reduzido, então se somado à redução promovida pela atividade física, melhoria da alimentação (alimentos menos gordurosos e calóricos, mais frutas e legumes, dentre outros), redução do estresse, consumo de sal, dentre outras medidas não medicamentosas, pode ser possível retirar medicamentos do tratamento com manutenção dos níveis pressóricos dentro dos limites considerados de normalidade.

Os medicamentos em uso não estão de acordo com os que são citados na literatura atualmente como os melhores na redução dos níveis pressóricos. Várias pesquisas indicam a 
soberania dos BRA na redução dos níveis pressóricos e também como os melhores em relação ao perfil de efeitos indesejáveis, no entanto, foi observado que os betabloqueadores foram os mais prescritos na amostra estudada. Em se tratando de idosos, os betabloqueadores podem não ser uma boa opção ao se considerar a maior prevalência de insuficiência cardíaca crônica (ICC) em tal grupo populacional, a menos que o betabloqueador em questão fosse o carvedilol, o bisoprolol ou o succinato de metoprolol, os quais são considerados comprovadamente eficazes nos quadros de ICC (BOCCHI et al., 2012). Porém, os resultados indicaram o atenolol como o betabloqueador mais prescrito, o qual é considerado o melhor de sua classe no controle dos níveis pressóricos.

A losartana não é considerada o melhor representante da classe, mas é a única disponível no serviço público. A hidroclorotiazida é o melhor diurético indicado no controle da HAS, muito frequentemente em associação, mas também com eficácia até mesmo como monoterapia em casos de HAS leve a moderada, uma vez que não é eficaz na redução dos níveis pressóricos em mais de $15 \mathrm{mmHg}$. Alguns poucos pacientes encontravam-se sob tratamento com furosemida, a qual apresenta maior indicação dentro de sua classe, em casos de HAS associada à insuficiência renal. Também a espironolactona foi observada em uso, sendo sua principal indicação em associação aos diuréticos depletores de potássio, o que foi observado na maioria das prescrições. No entanto, em um dos casos foi observada sua prescrição como monoterapia sendo tal indicação considerada mais apropriada em casos de hipertensão arterial associada ao hiperaldosteronismo (FERNANDES et al., 2016).

Ao se considerar a monoterapia, os IECA foram os mais prescritos, porém com pouca diferença em relação à prescrição dos $\mathrm{BRA}$ e dos $\mathrm{BB}$. Ainda em relação à monoterapia, o anlodipino foi o mais prescrito dentre os BCC e tal resultado está de acordo com as características de tal medicamento pertencente à classe das di-hidropiridinas, as quais estão frequentemente associadas a quadros de taquicardia reflexa quando em monoterapia. $\mathrm{O}$ anlodipino, no entanto, apresenta menor potencial em causar taquicardia reflexa.

Cerca de $1 / 3$ dos pacientes sob tratamento com anti-hipertensivos e quase $50 \%$ dos pacientes sob tratamento com dois anti-hipertensivos não apresentavam sua PA controlada, dado este preocupante, uma vez que mesmo sob tratamento e, exposto aos seus efeitos colaterais, assim como apresentando gastos pessoais ou para o serviço público de saúde, o paciente não se encontra protegido do agravamento do quadro e, com potenciais lesões em órgãos-alvo. Assim, torna-se premente a necessidade de melhor acompanhamento dos pacientes hipertensos a fim de se avaliar a resposta à terapia com medicamentos anti-hipertensivos e detectar os principais problemas relacionados, prevenindo-se a ocorrência de crises hipertensivas bem como de lesões em órgãosalvo. A maioria dos pacientes não relatou problemas quanto a efeitos colaterais importantes dos medicamentos anti-hipertensivos.

\section{Conclusões}

De acordo com a metodologia aplicada pode-se concluir que os medicamentos em uso no tratamento e controle da HAS não são aqueles considerados mais eficazes atualmente e um número expressivo de pacientes sob tratamento não apresenta os níveis pressóricos dentro dos limites de normalidade, em especial aqueles sob tratamento com dois medicamentos anti-hipertensivos. Assim, a pesquisa deixa clara a necessidade de melhor acompanhamento dos profissionais de saúde quanto à resposta à terapia medicamentosa e não medicamentosa a fim de que se possa realmente prevenir danos maiores aos pacientes.

\section{Referências}

ANDRADE, J.P.; VILAS-BOAS, F.; CHAGAS, H. et al. Aspectos epidemiológicos da aderência ao tratamento da hipertensão arterial sistêmica. Arq Bras Cardiol., v. 79, n. 4, p. 375-383, 2002. 
BARROS, M.B.A.; FRANCISCO, P.M.S.B.; LIMA, M.G. et al. Social inequalities in health among the elderly. Cad Saúde Pública, v. 27, n. 2, p. 198-208, 2011.

BRASIL. Ministério da Saúde - Conselho Nacional de Saúde. 2013. Resolução n. 466, de 12 de dezembro de 2012 - Normas regulamentadoras de pesquisas envolvendo seres humanos. Publicada em 13/06/2013. Disponível em: http://conselho.saude.gov.br/resolucoes/2012/Reso466.pdf Acesso em 02 de abril de 2015.

BOCCHI, E.A.; MARCONDES-BRAGA, F.G.; BACAL, F. et al. Sociedade Brasileira de Cardiologia. Atualização da Diretriz Brasileira de Insuficiência Cardíaca Crônica - 2012. Arq Bras Cardiol., v. 98, n. 1, p. 1-33, 2012.

BRITO VIEIRA, C.P.; NASCIMENTO, J.J.; BARROS, S.S. et al. Prevalência referida, fatores de risco e controle da hipertensão arterial em idosos. Cienc Cuid Saude, v. 15, n. 3, p. 413-420, 2016.

CARVALHO, M.V. de; SIQUEIRA, L.B.; SOUSA, A.L.L. et al. A Influência da Hipertensão Arterial na qualidade de vida. Arquivo Brasileiro Cardiologia, v. 100, n. 2, p.164-174, 2013.

DUARTE, O. de O.; FARIA, W.R. de C.; PINTO, F.M. et al. Tratamento ambulatorial da hipertensão arterial sistêmica - Revisão de literatura. Revista UNINGÁ, v. 17, n. 2, p. 22-29, 2014.

FERNANDES, V.; SILVA, T.; MARTINS, D. et al. Hiperaldosteronismo primário: resultados do primeiro estudo multicêntrico português realizado pelo Grupo de Estudos de Tumores da SupraRenal. Revista Portuguesa de Endocrinologia, Diabetes e Metabolismo, v. 11, n. 2, p. 163170, 2016.

GIROTTO, E.; ANDRADE, S.M.; CABRERA, M.A.S. et al. Adesão ao tratamento farmacológico e não farmacológico e fatores associados na atenção primária da hipertensão arterial. Ciênc Saúde Coletiva, v. 18, n. 6, p. 1763-1772, 2013.

GUSMAO, J.L. de; MION JR, D. Adesão ao tratamento - conceitos. Rev. Bras.Hipertens, v. 13, n. 1, p. 23-25, 2006.

IBRAHIM, M.M.; DAMASCENO, A. Hypertension in developing countries. Lancet, v. 380, n. 9841, p. 611-9, 2012. DOI:10.1016/S0140-6736(12)60861-7

MALACHIAS, M.V.B.; SOUZA, W.K.S.B.; PLAVNIK, F.L. et al. VII Diretriz Brasileira de Hipertensão Arterial. Arq Bras Cardiol., v. 107, n. 3, 83 p., 2016.

MENEZES, M.H.; REIS, V.H.S.; DANTAS, D.B.G. et al. Hipertensão arterial sistêmica e eventos cardiovasculares no Estado do Tocantins, Brasil. Revista de Patologia do Tocantins, v. 4, n. 2, p. 50-53, 2017.

PIMENTA, F.B.; PINHO, L.; SILVEIRA, M.F. et al. Fatores associados a doenças crônicas em idosos atendidos pela Estratégia de Saúde da Família. Ciênc Saúde Coletiva, v. 20, n. 8, p. 248998, 2015. 
REIS, A.F.N.; CESARINO, C.B. Fatores de risco e complicações em usuários cadastrados no Hiperdia de São José do Rio Preto. Cienc Cuid Saúde, v. 15, n. 1, p. 118-24, 2014.

SOCIEDADE BRASILEIRA DE CARDIOLOGIA; SOCIEDADE BRASILEIRA DE HIPERTENSÃO; SOCIEDADE BRASILEIRA DE NEFROLOGIA. VI Diretrizes Brasileiras de Hipertensão. Arquivos Brasileiros de Cardiologia, São Paulo, v. 95, n. 1, 58 p., 2010.

SUDRÉ, M.R.S.; REINERS, A.A.O;; AZEVEDO, R.C.S. et al. Características socioeconômicas e de saúde de idosos assistidos pelas equipes de saúde da família. Cienc Cuid Saúde, v. 14, n. 1, p. 933-40, 2014. 